\title{
The first Japanese case of leukodystrophy with ovarian failure arising from novel compound heterozygous AARS2 mutations
}

\section{$\operatorname{AUTHOR}(\mathrm{S}):$}

Hamatani, Mio; Jingami, Naoto; Tsurusaki, Yoshinori; Shimada, Shino; Shimojima, Keiko; Asada-Utsugi, Megumi; Yoshinaga, Kenji; ... Takahashi, Ryosuke; Matsumoto, Naomichi; Yamamoto, Toshiyuki

\section{CITATION:}

Hamatani, Mio ... [et al]. The first Japanese case of leukodystrophy with ovarian failure arising from novel compound heterozygous AARS2 mutations. Journal of human genetics 2016, 61(10): 899-902

\section{ISSUE DATE:}

2016-10

URL:

http://hdl.handle.net/2433/217351

\section{RIGHT:}

This is the accepted version of the article, which has been published in final form at

http://dx.doi.org/10.1038/jhg.2016.64; The full-text file will be made open to the public on 02 December 2016 in

accordance with publisher's 'Terms and Conditions for Self-Archiving'; この論文は出版社版でありません。引用の際に は出版社版をご確認ざ利用ください。; This is not the published version. Please cite only the published version. 
1 Title: The First Japanese Case of Leukodystrophy with Ovarian Failure Arising from Novel

2 Compound Heterozygous AARS2 Mutations.

3

4 Contributors: Mio Hamatani ${ }^{1^{*}}$, Naoto Jingami ${ }^{1^{*}}$, Yoshinori Tsurusaki ${ }^{2}$, Shino Shimada ${ }^{3}$, Keiko

$5 \quad$ Shimojima ${ }^{3}$, Megumi Asada-Utsugi ${ }^{1}$, Kenji Yoshinaga ${ }^{1}$, Norihito Uemura ${ }^{1}$, Hirofumi

6 Yamashita ${ }^{1}$, Kengo Uemura ${ }^{1,4}$, Ryosuke Takahashi ${ }^{1}$, Naomichi Matsumoto ${ }^{2}$, Toshiyuki

7 Yamamoto $^{3}$

8

$9 \quad{ }^{1}$ Department of Neurology, Kyoto University Graduate School of Medicine, Kyoto, Japan

$10{ }^{2}$ Department of Human Genetics, Yokohama City University Graduate School of Medicine,

11 Yokohama, Japan

$12{ }^{3}$ Tokyo Women's Medical University Institute for Integrated Medical Sciences, Tokyo, Japan

$13{ }^{4}$ Department of Neurology, Ishiki Hospital, Kagoshima, Japan

14 *These authors contributed equally to this work.

Correspondence: Dr. H. Yamashita, Department of Neurology, Kyoto University Graduate School of Medicine, 54 Shogoin-Kawaharacho, Sakyoku, Kyoto 606-8507, Japan. Phone: +81-75-751-3771, Fax: +81-75-751-3265,

19 E-mail: hirofumi@kuhp.kyoto-u.ac.jp

20 Or Dr. K. Uemura, Department of Neurology, Ishiki Hospital, 2-4-15 Shimoishiki, Kagoshima 890-0005, Japan.

22 Phone: +81-99-220-4645, Fax: +81-99-220-4648,

23 E-mail: ueken@kuhp.kyoto-u.ac.jp

Running title: The First Japanese Case of AARS2 Mutation

Abbreviations: AARS2; alanyl-tRNA synthetase 2

Type of article: Short communication

Number of total pages: 14

Number of words (abstract): 98

Number of references: 17

Number of tables: 0 
Mio Hamatani \#2

1 Supplementary information: 1, Microsoft Word documents (.doc) 
Mio Hamatani \#3

\section{$1 \quad$ Abstract}

2 Even now, only a portion of leukodystrophy patients are correctly diagnosed, though

3 various causative genes have been identified. In the present report, we describe a case of

4 adult-onset leukodystrophy in a woman with ovarian failure. By whole exome sequencing, a

5 compound heterozygous mutation consisting of NM_020745.3 (AARS2_v001):c.1145C>A and

6 NM_020745.3 (AARS2_v001):c.2255+1G>A was identified. Neither of the mutations has been

7 previously reported, and this is the first report of AARS2 mutation in Asia. We anticipate that

8 further studies of the molecular basis of leukodystrophy will provide insight into its

9 pathogenesis and hopefully lead to sophisticated diagnostic and treatment strategies.

10 Key words: $A A R S 2 /$ compound heterozygous mutation/leukodystrophy/ovarian failure 
1

2

3

4

5

6

7

\section{Introduction}

Leukodystrophy is a syndrome with progressive white matter degeneration. Its pathogenic background and clinical presentation are variable. Genetic rather than environmental factors are associated with its variablity. ${ }^{1}$ More than a hundred subtypes of leukodystrophy have been reported, and many causative genes have been identified. ${ }^{2}$ However, the heterogeneity and complexity of leukodystrophy make a definitive diagnosis difficult. Indeed, only half of leukodystrophy patients receive a specific diagnosis. ${ }^{3}$

Here, we report a Japanese woman presenting with adult-onset leukodystrophy and ovarian failure in her thirties. By whole exome sequencing (WES), we detected novel compound heterozygous mutations in alanyl-transfer RNA (tRNA) synthetase 2 (AARS2) (OMIM *612035).

\section{Case report}

A 31-year-old Japanese woman first visited our hospital in 2010. She was born healthy with a normal delivery to non-consanguineous parents. She developed normally and graduated from college. She worked until she was 30 years old, when she suddenly quit her job and had reduced interactions with her surroundings. She developed cognitive decline and began abnormal behaviors (e.g., buying the same thing on consecutive days). She had difficulty using 
Mio Hamatani \#5

1 her left limbs, and she tended to lean to the left while sitting. She became totally incontinent. No

2 similar symptoms occurred in her parents or her older sister.

3 A general examination at age 33 revealed secondary amenorrhea, although she had normal

4 menstrual cycles until then with normal secondary sexual characteristics. The unelevated

5 gonadotropin level indicated the impairment of reactive gonadotropin release by hypothalamus

6 and pituitary, suggesting the secondary ovarian failure. She also had generalized atopic

7 dermatitis.

8 Neurological examination revealed significant decline in frontal lobe function. The patient

9 had a Mini-Mental State Examination score of 19/30 and a Frontal Assessment Battery score of

10 4/18. Left hand ideomotor apraxia, hyperreflexia, and rigidity of neck and limbs were observed

11 more on the left side. At the most recent examination at age 38, the patient was aphasic and

almost bedridden.

Brain magnetic resonance imaging (MRI) showed diffuse cerebral white matter

abnormalities without gadolinium enhancement. Patchy, diffusion-restricted areas were present in the abnormal white matter. Thinning of corpus callosum was also observed (Figure 1).

16 Cardiac ultrasound and electrocardiography showed no abnormalities. 
Mio Hamatani \#6

1 Gene analyses of colony stimulating factor 1 receptor $(C S F 1 R)$ and eukaryotic translation

2 initiation factor 2B (EIF2B) were normal. We performed WES on genomic DNA.

\section{Genetic analysis}

6 Committee, and written informed consent was obtained from the patient's parents. DNA

7 samples from the patient and her parents were analyzed by WES, as previously described. ${ }^{4}$

8 Detailed methods of WES and prioritization of variants are described in the supplementary

9 information.

We focused on de novo and recessive mutations. We found novel compound heterozygous mutations in AARS2, NM_020745.3 (AARS2_v001):c.1145C>A and NM_020745.3

(AARS2_v001):c.2255+1G>A. These were the missense mutation (p.T382K) and the splice-site mutation, respectively. These two mutations were not registered in the dbSNP137, NHLBI-ESP

6500, HGVD, or our in-house 575 Japanese control exome databases. Sanger sequencing confirmed that the mutations were transmitted maternally and paternally, respectively (Figure 2), and the in silico analysis for the pathogenicities is described in the supplementary information. 
1

2 heterozygous at the locus for c.1145, and the sequence around c. 2255 was normal without any

3 aberrant insertion or truncation (Figure 3c).

splice-site mutation (Figure 3b). Direct sequence analysis of cDNA revealed that the patient was

6

\section{Discussion}

The causative gene in a Japanese leukodystrophy patient with ovarian failure was identified

(1)

as a compound heterozygous AARS2 mutation.

We initially considered the patient's diagnosis as hereditary diffuse leukoencephalopathy

with axonal spheroids, which is most prevalent in women in their 30s and 40s and frequently

includes corpus callosum thinning. ${ }^{5-7}$ We also suspected vanishing white matter disease, which

includes ovarian failure as one of its features. ${ }^{8-11}$ However, no mutation in causative genes for

these diseases were identified.

Therefore, we performed a comprehensive genetic workup and, by use of WES, compound heterozygous mutations, c. 1145C>A (p.T382K) and c.2255+1G>A, were detected. These

mutations were inherited as an autosomal recessive trait. Both mutations were novel and

predicted as pathogenic in silico. In the analysis of the patient's mRNA, we could not detect any

misspliced mature mRNA caused by the splice-site mutation, c. $2255+1 \mathrm{G}>\mathrm{A}$. However, it is

predicted that the mutation introduced a premature stop codon when the splicing at the 
exon16-intron 16 boundary failed (Figure $3 a$ ). Then this transcript would be a target for the

2 nonsense-mediated mRNA decay and should be degraded. mitochondrial dysfunction and reported in 6 patients with adult-onset leukodystrophy ${ }^{12}$. In that report, two specific clinical phenotypes were described: characteristic MRI features and ovarian

7 failure. Common MRI features are striking white matter tract involvement and presence of spots

8 of restricted diffusion in the cerebral white matter. Corpus callosum thinning and the strip of

9 signal abnormality in the splenium were also seen. Ovarian failure was present in all of the

previously reported 5 female cases; 1 case had secondary amenorrhea like the present patient,

11 and 4 cases had primary amenorrhea.

AARS2 mutations have another subtype, severe infantile cardiomyopathy with early fatal

outcome. ${ }^{13-15}$ In contrast to this infantile subtype, the later-onset subtype, which includes the

present case, has no signs of cardiomyopathy. Therefore, the question arises why AARS2 
infantile-onset cardiomyopathy had the c.1774C $>\mathrm{T}$ (p.R592W) mutation located in the editing

2 domain in at least one allele, which severely compromises aminoacylation. On the other hand,

3 none of the missense mutations in adult-onset leukodystrophy patients were in the editing

4 domain, while at least one missense mutation was in the aminoacylation domain, leading to

5 relatively partial reduction in aminoacylation activity. The location of the compound

6 heterozygous mutations in the present case is consistent with the theory.

$7 \quad$ In conclusion, we describe a Japanese woman with novel compound heterozygous

8 mutations in $A A R S 2$, the first report of leukodystrophy caused by AARS2 mutations in Asia.

9 This is also the subsequent report after the first description in 2014 for 6 adult-onset

10 leukodystrophy patients by AARS2 mutations. All reported cases including the present case are

11 apparently sporadic without family history and caused by compound heterozygous mutations,

making the correct diagnosis very difficult. Therefore, if you will notice the characteristic MRI

findings and, especially in female, the existence of ovarian failure, you should examine the

$A A R S 2$ gene. 
Mio Hamatani\#10

1

2 Acknowledgement

3 This study was supported by a Grant-in-Aid of Health Labor Sciences Research Grants from the

4 Ministry of Health, Labor and Welfare, Japan (T.Y., H.Y. and N.M.). This study was also

5 supported by the Japan Society for the Promotion of Science (Grants-in-Aid for Scientific

6 Research (A) and (C)); the fund for the Creation of Innovation Centers for Advanced

7 Interdisciplinary Research Areas Program in the Project for Developing Innovation Systems

8 from the Japan Science and Technology Agency; a Grant-in-Aid for Scientific Research on

9 Innovative Areas (Transcription Cycle) from the Ministry of Education, Culture, Sports, Science

10 and Technology of Japan; the Takeda Science Foundation; and the Strategic Research Program

11 for Brain Science from Japan Agency for Medical Research and Development (N.M.).

\section{Conflict of Interest}

14 The authors declare no conflict of interest.

16 Supplementary information accompanies the paper on Journal of Human Genetics website. 
References

Kaye EM, Moser H. Where has all the white matter gone? Unraveling the mysteries of leukoencephalopathies. Neurology. 2004;62:1464-5.

2. Renaud DL. Inherited leukoencephalopathies. Semin Neurol. 2012;32:3-8.

3. Schiffmann R, van der Knaap MS. Invited article: an MRI-based approach to the diagnosis of white matter disorders. Neurology. 2009;72:750-9.

4. Tsurusaki Y, Koshimizu E, Ohashi H, Phadke S, Kou I, Shiina M, et al. De novo SOX11 mutations cause Coffin-Siris syndrome. Nat Commun. 2014;5:4011. 5. Rademakers R, Baker M, Nicholson AM, Rutherford NJ, Finch N, Soto-Ortolaza A, et al. Mutations in the colony stimulating factor 1 receptor (CSF1R) gene cause hereditary diffuse leukoencephalopathy with spheroids. Nat Genet. 2012;44:200-5. 6. Nicholson AM, Baker MC, Finch NA, Rutherford NJ, Wider C, Graff-Radford NR, et al. CSF1R mutations link POLD and HDLS as a single disease entity. Neurology. 2013;80:1033-40.

7. Konno T, Tada M, Koyama A, Nozaki H, Harigaya Y, Nishimiya J, et al. Haploinsufficiency of CSF-1R and clinicopathologic characterization in patients with HDLS. Neurology. 2014;82:139-48. 8. van der Knaap MS, Kamphorst W, Barth PG, Kraaijeveld CL, Gut E, Valk J. Phenotypic variation in leukoencephalopathy with vanishing white matter. Neurology. 1998;51:540-7.

9. Fogli A, Gauthier-Barichard F, Schiffmann R, Vanderhoof VH, Bakalov VK, Nelson LM, et al. Screening for known mutations in EIF2B genes in a large panel of patients with premature ovarian failure. BMC Womens Health. 2004;4:8. 10. Mathis S, Scheper GC, Baumann N, Petit E, Gil R, van der Knaap MS, et al. The ovarioleukodystrophy. Clin Neurol Neurosurg. 2008;110:1035-7.

11. Bugiani M, Boor I, Powers JM, Scheper GC, van der Knaap MS.

Leukoencephalopathy with vanishing white matter: a review. J Neuropathol Exp Neurol. 2010;69:987-96.
12.
Dallabona C, Diodato
D, Kevelam SH, Haack TB, Wong LJ, Salomons GS, et al. Novel (ovario) leukodystrophy related to AARS2 mutations. Neurology. 2014;82:2063-71. 13. Gotz A, Tyynismaa H, Euro L, Ellonen P, Hyotylainen T, Ojala T, et al. Exome sequencing identifies mitochondrial alanyl-tRNA synthetase mutations in infantile mitochondrial cardiomyopathy. Am J Hum Genet. 2011;88:635-42.

14. Taylor RW, Pyle A, Griffin H, Blakely EL, Duff J, He L, et al. Use of whole-exome sequencing to determine the genetic basis of multiple mitochondrial respiratory chain complex 
1 deficiencies. JAMA. 2014;312:68-77.

2 15. Calvo SE, Compton AG, Hershman SG, Lim SC, Lieber DS, Tucker EJ, et al.

3 Molecular diagnosis of infantile mitochondrial disease with targeted next-generation sequencing.

$4 \quad$ Sci Transl Med. 2012;4:118ra10.

$5 \quad$ 16. Euro L, Konovalova S, Asin-Cayuela J, Tulinius M, Griffin H, Horvath R, et al.

6 Structural modeling of tissue-specific mitochondrial alanyl-tRNA synthetase (AARS2) defects

7 predicts differential effects on aminoacylation. Front Genet. 2015;6:21.

8 17. van Berge L, Hamilton EM, Linnankivi T, Uziel G, Steenweg ME, Isohanni P, et al.

9 Leukoencephalopathy with brainstem and spinal cord involvement and lactate elevation: clinical

10 and genetic characterization and target for therapy. Brain. 2014;137:1019-29. 
$1 \quad$ Titles and legends to figures

2 Figure 1. Brain MRI of the patient at age 33

3 The fluid attenuated inversion recovery (FLAIR) images show periventricular white matter

4 abnormalities predominant at the parietal and frontal area, which revealed low signal lesions in

5 the T1-weighted image (arrows in images $\mathrm{a}, \mathrm{b}$ and $\mathrm{c}$ ). The diffusion-weighted image shows

6 patchy areas of restricted diffusion in the abnormal white matter, which are confirmed by low

7 signal of the corresponding areas on the apparent diffusion coefficient map (arrows in image d,

8 e and f). The sagittal FLAIR image shows thinned corpus callosum, especially in the splenium

9 (arrow in image g) and affected white matter structures in a tract-like manner (arrow in image h).

10 The MR angiography showed no stenosis of the major vessels (image i).

Figure 2. Sanger sequencing of the patient and her parents

The mutations were confirmed by Sanger sequencing. c.1145C>A (p.T382K) was transmitted

maternally, and c. $2255+1 \mathrm{G}>\mathrm{A}$ was transmitted paternally.

Figure 3. mRNA analysis

(a) Genomic structure of AARS2 was illustrated. Arrowheads indicate primer pairs. Triplets 
Mio Hamatani\#14

1 splicing error, a premature stop codon will appear just afterwards (blue). (b) Image of PCR

2 products of cDNA including each mutation (PCR1 and PCR2). (c) Direct sequence analysis of

3 patient's cDNA. 

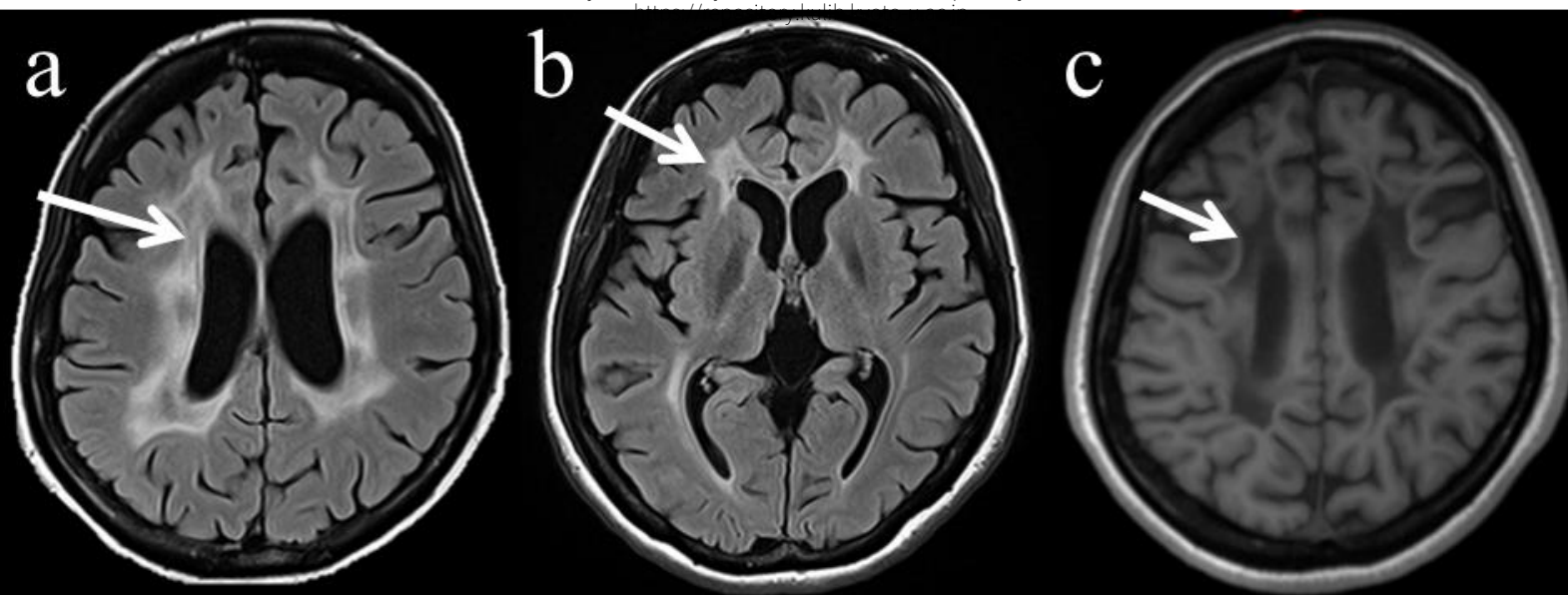

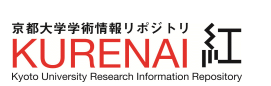

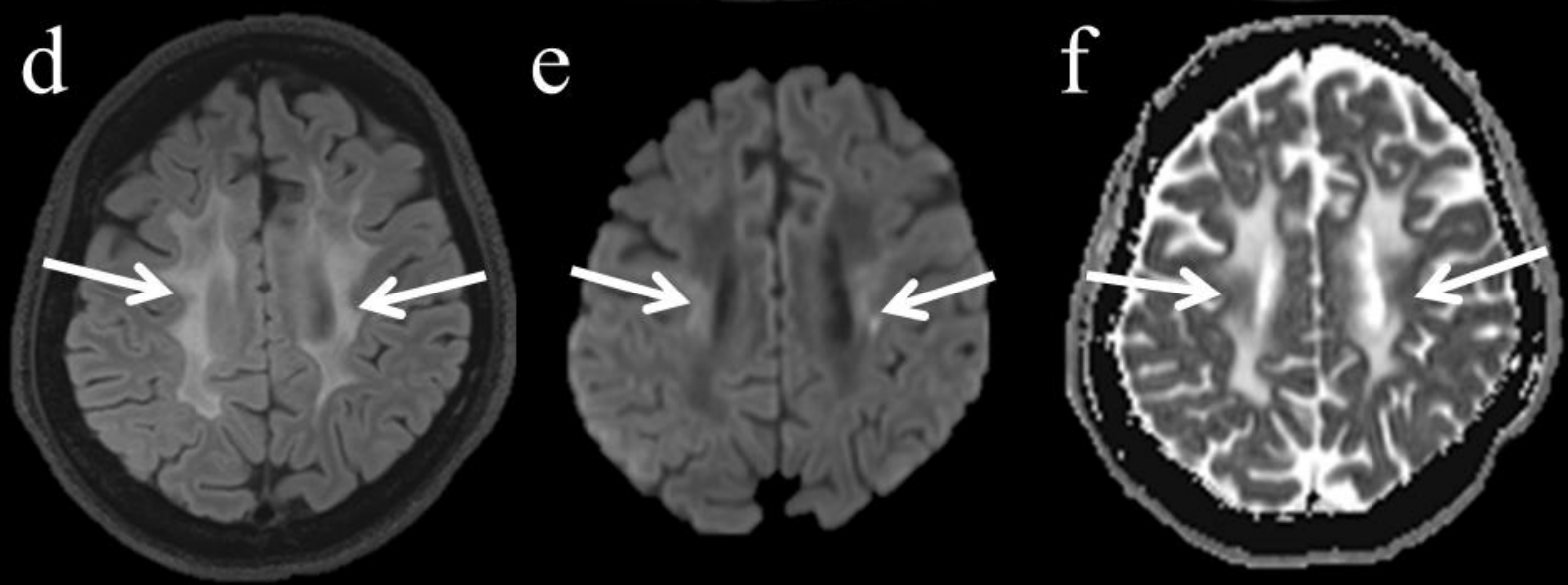

Figure 1

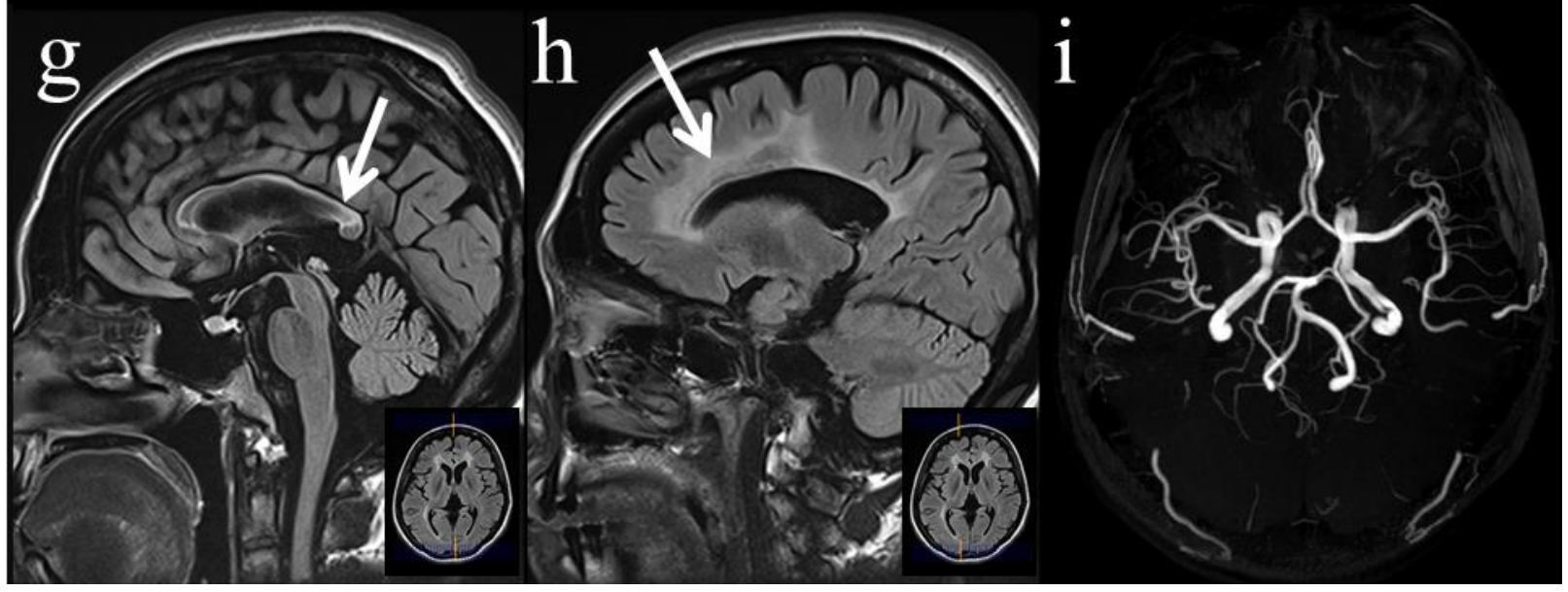




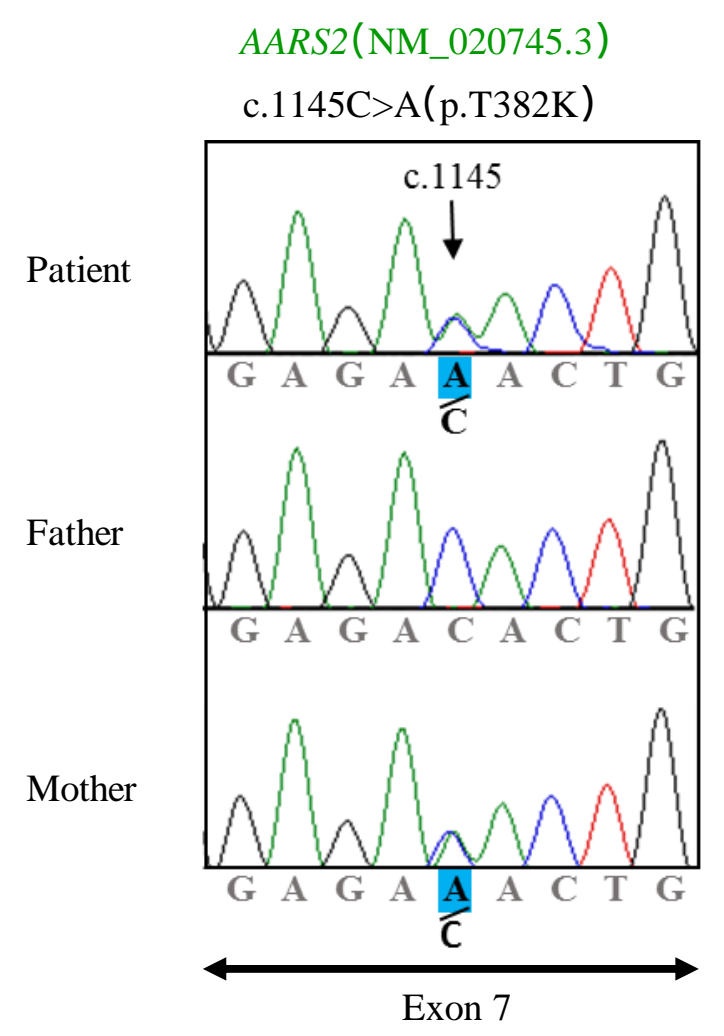

Maternal inheritance
AARS2(NM_020745.3)

c. $2255+1 \mathrm{G}>\mathrm{A}$

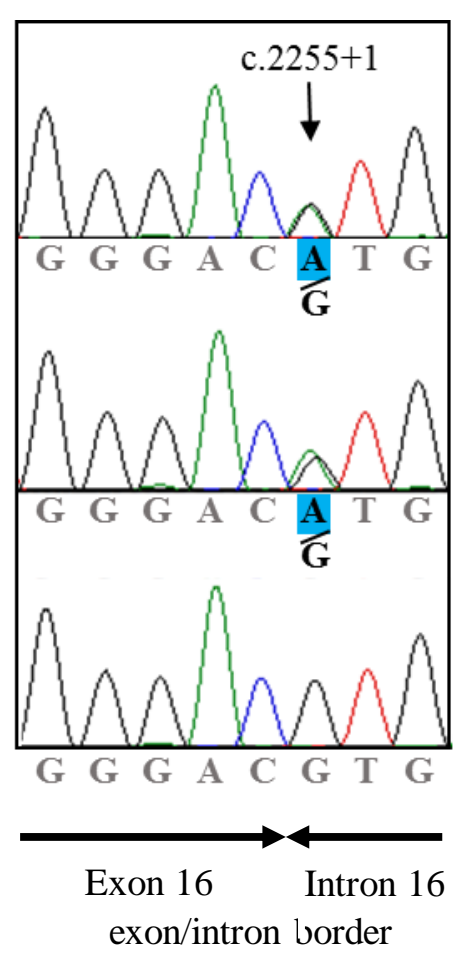

Paternal inheritance

Figure 2 
a

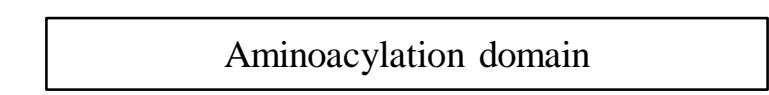

PCR1

$511 \mathrm{bp}$ (Amplicon of mRNA)

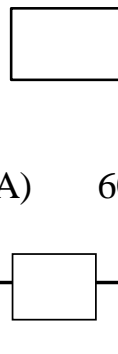

Ex7

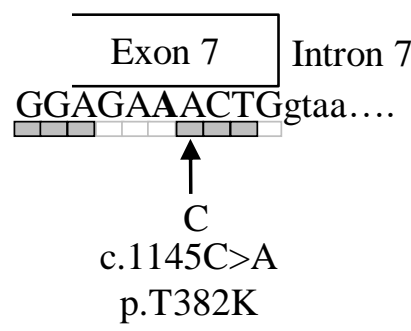

p.T382K

C

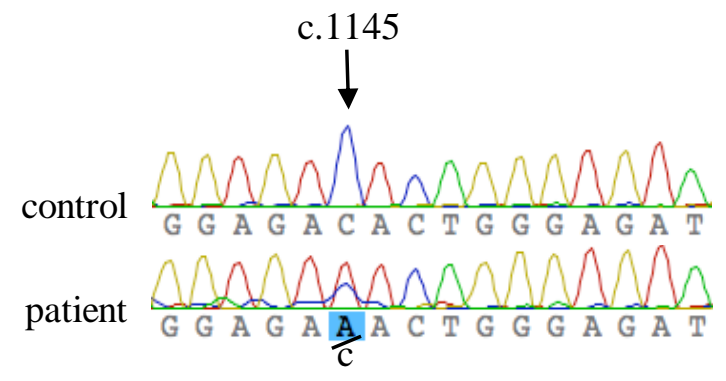

\begin{tabular}{l|l}
\hline Exon 7 & Exon 8 \\
\hline
\end{tabular}

\section{Editing domain}

\section{PCR2}

$600 \mathrm{bp}$ (Amplicon of mRNA)

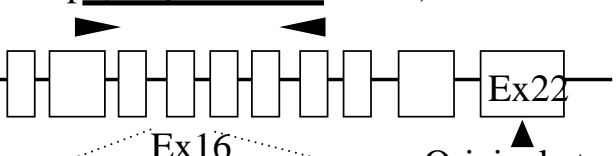

Original stop codon

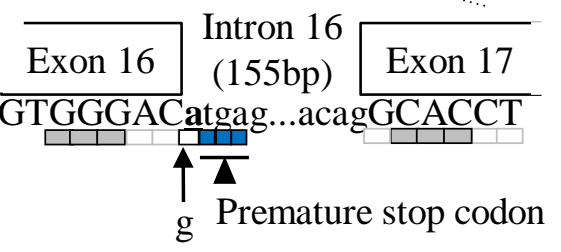

c. $2255+1 \mathrm{G}>\mathrm{A}$

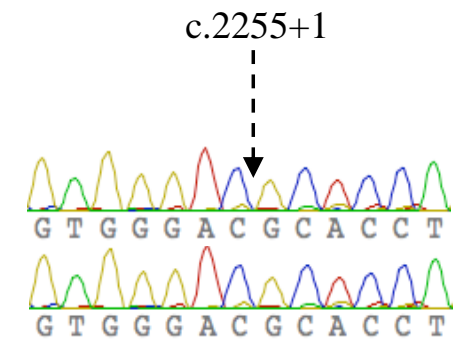

Exon 16 b

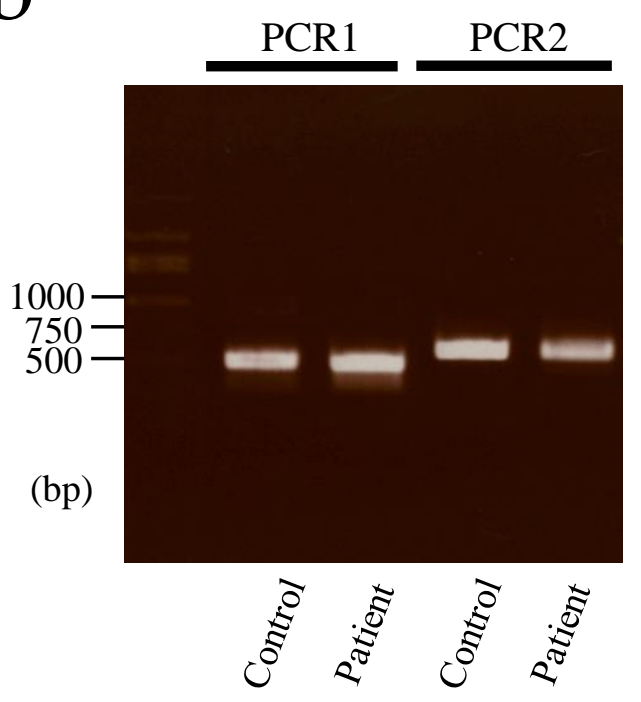

\section{Figure 3}




\section{Supplementary information}

\section{Whole exome sequencing and in silico analysis for the pathogenicities}

DNA samples from the patient and her parents were analyzed by whole exome sequencing (WES) as previously described. Genomic DNA was captured by using the SureSelect Human All Exon v5 $(50 \mathrm{Mb})$ Kit (Agilent Technologies, Santa Clara, CA). Captured DNA was sequenced on a HiSeq2500 (Illumina, San Diego, CA) with 101 bp paired-end reads and 7 bp index reads. The mean read depth against RefSeq coding sequence (CDS) was 38.18-158.75 reads with 74.6-95.5\% of CDS being covered by 20 or more reads. Image analysis and base calling were performed by sequence control software real-time analysis and CASAVA software (v1.8) (Illumina). The quality controlled reads were mapped to the human reference genome (UCSC hg19, NCBI build 37.1) by using Novoalign (v3.00.02). After the removal of PCR duplication by using Picard (v1.55), single nucleotide variants (SNVs) and short insertions and deletions (Indels) were identified by using Genome Analysis Toolkit (GATK) (v1.6-5) and annotated by using ANNOVAR (2013 jun).

Out of all variants within exons and regions $\pm 30 \mathrm{bp}$ from the exon-intron boundaries, those registered common single nucleotide polymorphisms (SNP) with minor allele frequency of $>1 \%$ in dbSNP137, and common SNP containing six or more individuals in our in-house database (exome data from 575 Japanese individuals), and synonymous variants were all removed. The variants were confirmed by Sanger sequencing with an ABI PRISM 3500xl or ABI3130xl autosequencer (Life Technologies, Carlsbad, CA).

We focused on de novo and recessive mutations, then we found novel compound heterozygous mutations in AARS2.The missense mutation (p.T382K) was predicted as deleterious (0.01) by SIFT (http://sift.jcvi.org/), possibly damaging (0.712) by Polyphen-2

(http://genetics.bwh.harvard.edu/pph2/). The splice-site mutation (c.2255+1G>A) was predicted as abolishing a donor site by BDGP (http://www.fruitfly.org/seq_tools/splice.html) and NetGene2 (http://www.cbs.dtu.dk/services/NetGene2/), and as altering the donor site and most probably affecting splicing (86.07 to 59.23) by Human Splicing Finder (http://www.umd.be/HSF3/).

\section{mRNA analysis}

Total RNA was extracted from the white blood cells of the patient and a healthy control by using QIAamp RNA Blood Mini and RNase-Free DNase set (QIAGEN). cDNA was synthesized with random primers by use of First-Strand cDNA Synthesis Kit (GE Healthcare). cDNA of AARS2 was amplified by polymerase chain reaction (PCR) using the primer pairs,

5'-GCCGCCTTTCTGAACTTCTT-3', and 5'-GGCAGCTTCCAGGTCAGTAG-3'. Then, segments harboring c.1145C>A or c.2255+1G>A were amplified by nested-PCR using the primer pairs, 5'-CTGCAAGGCAAACACTCCAC-3' and 5'-CCAGGTCTCCACACAGTGAC-3', 5'-AGGGCTCCCATCTCAATCCT-3' and 5'-GCTGCAGCATCTTCACTGTG-3', respectively (Figure 3a). 